\title{
Pushing performances of nonlinear optics in silicon nitride waveguides
}

\author{
Camille-Sophie Brès, Davide Grassani \\ Ecole Polytechnique Fédérale de Lausanne, Photonic Systems Laboratory (PHOSL), STI-IEL, Station 11, CH-1015 \\ Lausanne, Switzerland \\ camille.bres@epfl.ch
}

\begin{abstract}
Silicon nitride waveguides offer excellent linear and nonlinear properties that can be exploited from the visible to the middle infrared. Here we will cover recent efforts on pushing performances of $3^{\text {rd }}$ and $2^{\text {nd }}$ order nonlinear wave mixing in such waveguides. (c) 2018 The Author(s)

OCIS codes: (190.4390) Nonlinear optics, integrated optics, (130.3120) Integrated optics materials, (190.2620) Harmonic generation and mixing
\end{abstract}

\section{Background}

Integrated photonics has enabled the study and demonstration of many nonlinear effects thanks to the high optical confinement and the potential large nonlinear index of many integratable materials. Such platform could enable decreasing the required pump power for the design of more practical devices. There is a particular interest in unleashing the nonlinear optical performances of CMOS fabrication compatible materials, to take advantage of high quality fabrication capabilities and potential low cost mass production. Among various materials, silicon nitride $\left(\mathrm{Si}_{3} \mathrm{~N}_{4}\right)$ is one of the very interesting candidate and is often praised for its relatively high nonlinear index, its low linear loss, its wide transparency window and its nearly non-existant nonlinear losses at telecom wavelengths. As such many advances in fabrication and impressive experimental demonstrations have recently been made. In particular, large cross sections waveguide can now be fabricated [1], while the control of exact material composition can be adjusted to tune bandgap and other properties (nonlinearity, refractive index, loss) [2]. High quality waveguides and microrings have been successfully used for the generation of supercontinuum (SC) and Kerr frequency combs [3]. The development of such SC sources using photonic chips is indeed attracting significant research attention as this platform could support direct on-chip generation towards the middle infrared (MIR). MIR SC generation, of particular importance for spectroscopy, is being extensively studied. However driving the process from compact femto-second fiber laser in the simplest configuration possible could provide a solution towards compact, portable and robust devices.

In addition to these $\chi^{(3)}$ phenomena, the potential photonic integration of $\chi^{(2)}$ could unlock many other nonlinear effects. These are still lacking in the materials with the best photonic integration capabilities, as in the dipole approximation $\chi^{(2)}$ vanishes for centro-symmetric or amorphous materials. While weak native $\chi^{(2)}$, possibly attributed to interface effects or film stress induced during deposition, has been previously detected, the performance is still far behind that obtained from non-centrosymmetry materials. Overall, pushing the performance of both $\chi^{(3)}$ and $\chi^{(2)}$ nonlinear effects in silicon nitride could provide the efficiency and practicality thought after in such integrated devices.

\section{Recent advances}

CMOS integrated waveguides represent an excellent solution for SC generation. Owing to their high optical confinement and high nonlinear index, significant broadening with moderate pump powers directly obtained from fiber lasers have been demonstrated. Soliton induced dispersive wave (DW) generation is a mechanism that enables extending SCG. A DW has to be phased matched to the soliton pulse seeded by the pump. Typical material dispersion readily results in a blue-shifted phase matched wavelength with respect to the pump. It is however possible to shape the waveguide dispersion such that a second phased matched wavelength, this time red shifted towards the MIR, be obtained despite the very strong anomalous material dispersion [5]. Based on large cross-section waveguides, with low propagation loss below $1 \mathrm{~dB} / \mathrm{cm}$, we show that the DW phase matching point can be positioned and tuned within the functional group region $(2.5-4 \mu \mathrm{m})$ by lithographic control of the waveguide dimensions (Fig.1a). In Fig.1b, the DW generated from a 2 micron femto-second fiber laser is experimentally measured, in agreement with theory. The combination of large waveguide and $2 \mu \mathrm{m}$ pumping enables power conversion efficiencies as high as $30 \%$ [6].

Back in the 80's it was shown that material not normally exhibiting any second order nonlinearity, namely silica [7], can be all optically poled such that an effective $\chi^{(2)}$ be obtained from an induced permanent electric field and 
material $\chi^{(3)}$. Recently the same effect was observed in integrated $\mathrm{Si}_{3} \mathrm{~N}_{4}$ waveguide [8,9]. As a consequence, an initial weak second harmonic of an input signal quickly grows with time, as experimentally observed in Fig.2a, and reaching 3 orders of enhancement. As this effect is formed from coherent multiphoton absorption processes generating a photo-current with direction following a phase mismatch $\Delta \beta=\beta_{s h}-2 \beta_{p}$, with $\beta_{s h / p}$ the wave vector of the second harmonic/pump, the effective $\chi^{2}$ periodicity automatically adjusts to pumping conditions. This is illustrated in Fig.2a, where in the same waveguide similar SH growths are experimentally measured for different pump wavelengths. We also verified, that a phase matching peak appears near the poling pump wavelength and that the peak moves when the poling pump is changed. The significant enhancement enables the detection of SH signals on a silicon detector [10]. Before poling, the 200 ps pulses could not be detected. During the poling process, a growth of the SH pulses is observed on the oscilloscope resulting in a converted pulse train with excellent quality (Fig.2b and c).

(a)

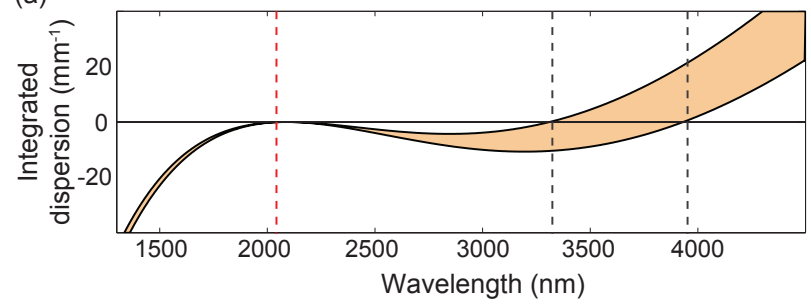

(b)

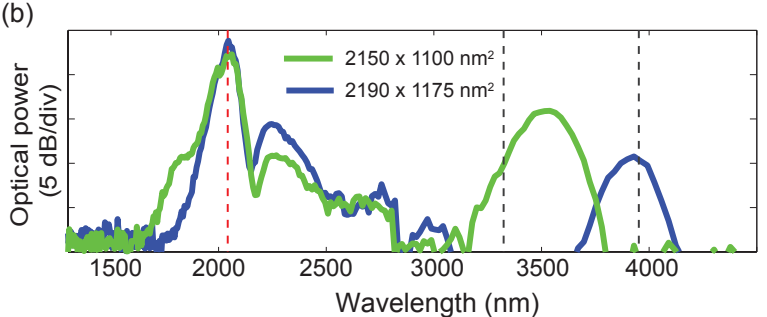

Fig. 1. (a) Simulated integrated dispersion for waveguide width between 1050-1175nm (height 2150nm). DW phase matching with $2090 \mathrm{~nm}$ pump (red line) is located in MIR. (b) Experimental result of SC generation in $5 \mathrm{~mm}$ long waveguides with different cross-sections for estimated pump power of $13.6 \mathrm{~mW}$

(a)

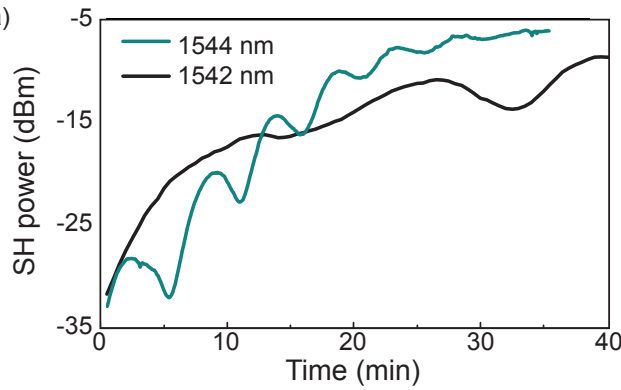

(b)

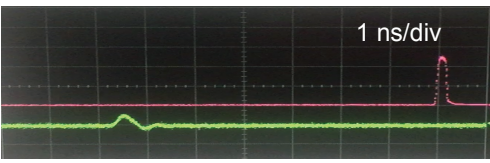

(c)

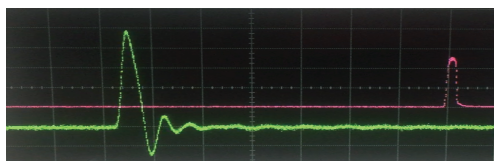

Fig. 2. (a)Growth of SH over time for constant pump ( $90 \mathrm{~W}$ peak) at 1542 or $1544 \mathrm{~nm}$ coupled into a $4 \mathrm{~cm}$ long SiN waveguide. Telecom (pink) and SH (green) pulse trains: (b) during poling and (c) after poling. Vertical axis: pink $25 \mathrm{mV} / \mathrm{div}$, green $5 \mathrm{mV} / \mathrm{div}$

We show how third order nonlinear optical nonlinearity in SiN can be exploited for MIR light generation and how all-optical poling can significantly increase the efficiency of second order nonlinearity. Work supported by the European Research Council (ERC-2012-StG 306630-MATISSE and 771647 PISSARRO).

1. C. Herkommer et al, "A chip-based silicon nitride platform for mid-infrared nonlinear photonics," in Conference on Lasers and Electro-Optics (CLEO) SM2K.6, (2017).

2. C.J. Krückel et al, “Optical bandgap engineering in nonlinear silicon nitride waveguides,”Opt. Express (25) 13, 15370 (2017).

3. X. Liu et al., "Octave-spanning supercontinuum generation in a silicon-rich nitride waveguide," Opt. Lett. (41) 12, 2719 (2016).

4. V. Brasch et al., "'Photonic chip based optical frequency comb using soliton induced Cherenkov radiation," Science (351), 357 (2016).

5. H. Guo et al., "Mid-infrared frequency comb via coherent dispersive wave generation in silicon nitride nanophotonic waveguides,"Nat. Photonics (12) 6, 330 (2018).

6. D. Grasanni et al., "Highly efficient 4 micron light generation through fs-fiber laser driven supercontinuum in $\mathrm{Si}_{3} \mathrm{~N}_{4}$ waveguides," arXiv preprint arXiv:1806.06633 (2018).

7. U. Österberg et al., "Dye laser pumped by Nd:YAG laser pulses frequency doubled in a glass optical fiber," Opt. Lett. (11), 516 (1986).

8. A. Billat et al., "Large second harmonic generation enhancement in $\mathrm{Si}_{3} \mathrm{~N}_{4}$ waveguides by all-optically induced quasi-phase-matching," Nat. Comm. (8) 1, 1016 (2017).

9. M.A.G. Porcel et al, "Photo-induced second-order nonlinearity in stoichiometric silicon nitride waveguides," Opt. Express (25), 33143 (2017).

10. D. Grassani et al, "Observation of Second Harmonic and Sum Frequency in an Optically Poled $\mathrm{Si}_{3} \mathrm{~N}_{4}$ Waveguide," in Conference on Lasers and Electro-Optics (CLEO), SF2A.2 (2018). 